\title{
Problematic issues in breast core needle biopsies
}

\author{
Stuart J. Schnitt ${ }^{1}$
}

Received: 19 July 2018 / Accepted: 22 July 2018 / Published online: 2 January 2019

(c) United States \& Canadian Academy of Pathology 2019

\begin{abstract}
Image-directed core needle biopsies of the breast are routinely used in current clinical practice for the initial assessment of non-palpable breast lesions. This article provides an update on several important issues regarding evaluation of breast core needle biopsies.
\end{abstract}

Core needle biopsy (CNB) using image-directed guidance methods, including stereotactic mammography, ultrasound, and magnetic resonance imaging (MRI), is now the standard of care for the initial evaluation of nonpalpable breast lesions [1-3]. This article will review some CNB basics, discuss several diagnostic pitfalls in the evaluation of CNBs (and how to avoid them), provide an update on the management of high risk lesions encountered in core needle biopsies, and describe the current status of ancillary biomarker studies and molecular testing in breast CNB.

\section{Core needle biopsy basics}

CNB of non-palpable breast lesions began in the late 1980s with several goals in mind including: (1) overcoming the limitations of fine needle aspiration for the evaluation of these lesions (including a high inadequacy rate and the inability to distinguish invasive from in situ carcinomas); (2) provide a pre-operative diagnosis of breast cancer; and (3) spare patients with benign lesions the need for surgical excision. As noted above, in current clinical practice CNB is now the standard of care for the initial evaluation of breast lesions and, in fact, is a quality metric assessed for the accreditation of breast care programs.

Stuart J. Schnitt

sschnitt@bwh.harvard.edu

1 Department of Pathology, Brigham and Women's Hospital and Harvard Medical School, Dana-Farber Cancer Institute, Boston, MA, USA
Breast CNB can be performed under a variety of radiologic guidance methods including stereotactic mammography, ultrasound, and MRI. Some CNB devices are spring-loaded and discharge a cutting needle into the breast tissue. Others employ vacuum assistance following needle insertion to draw tissue into the cutting chamber and to facilitate sample collection. The use of vacuum-assisted devices results in substantially larger specimens than spring-loaded devices. Another advantage of vacuum-assisted devices is that they permit collection of numerous, contiguous samples with a single needle insertion in contrast to the multiple insertions required to obtain samples using spring-loaded devices. At many institutions, vacuum-assisted devices are used to sample microcalcifications, whereas spring-loaded devices are used to sample mass lesions.

The pathology requisition that accompanies a CNB specimen should indicate the laterality and location of the targeted lesion, the indication for the biopsy (i.e., mass, microcalcifications, architectural distortion, non-mass enhancement on MRI, etc.), the radiologist's differential diagnosis, the level of radiologic suspicion (usually provided by means of the BIRADS category), the imageguidance method, size of needle used, and the number of cores obtained. If the indication for CNB is mammographic microcalcifications, a specimen radiograph should be obtained by the radiologist, and the samples with the calcifications submitted separately from those without calcifications to identify for the pathologist the cores containing the mammographic target. This specimen radiograph should ideally be available for the pathologist to review when examining the case. CNB specimens should be submitted in their entirety for microscopic evaluation. While there is no universal agreement about the number of levels that should be cut from blocks of CNB specimens, a sufficient number of levels should be cut to permit as complete a 
pathologic-radiographic correlation as possible. We routinely cut three levels from each block for initial evaluation, and additional levels are cut as necessary. It should be noted that in some cases, relatively subtle pathologic changes can explain the findings on imaging studies and should, therefore, not be overlooked or dismissed as non-specific or inconsequential. For example, clusters of apocrine cysts or fragments of cyst wall characterized by fibrous tissue with at least some epithelium along one edge can each explain the presence of a mass or density seen on a mammogram.

Radiologic-pathologic correlation is essential in every case. If the findings on the initial histologic sections do not account for the findings on the imaging studies every attempt should be made to resolve the discrepancy. However, not all discrepancies can be resolved by further pathologic evaluation of the CNB specimen and if this occurs, it should be noted in the final pathology report. For example, if the indication for CNB is a mass lesion and the CNB samples reveal only unremarkable breast tissue after multiple levels are examined, the final report should indicate that histologic findings of a mass-forming lesion are not identified and that clinical and radiographic correlation are advised. Regularly scheduled radiology-pathology correlation conferences are of great value to discuss cases with radiologic-pathologic discordance and to formulate a plan to resolve such discrepancies.

Unfortunately, some cases are nebulous with regard to radiologic-pathologic concordance. For example, while pseudoangiomatous stromal hyperplasia (PASH) may produce a mass-forming lesion, it is also commonly seen as an incidental microscopic finding in breast tissue removed for another reason [4]. Therefore, it is sometimes unclear whether PASH identified in a CNB accounts for a mass or mass-like lesion seen on imaging studies or if it is simply incidental and unrelated to the radiographically targeted lesion. A recent study suggested that "diffuse PASH" in a CNB (defined as PASH involving two or more adjacent lobules) was significantly more often associated with a mass lesion than focal PASH. Therefore, providing some indication of the extent of PASH in a CNB may be of value in achieving radiologic-pathologic correlation in such cases [5]. In addition, the pathologic correlates of some imaging findings, such as a developing density on a mammogram or non-mass enhancement on MRI are in some cases uncertain, and the presence or absence of radiologic-pathologic concordance in such cases often rests on clinical judgment.

\section{Diagnostic pitfalls in core needle biopsies (and how to avoid them)}

The differential diagnostic problems encountered in CNB specimens largely mirror those seen in surgical breast specimens, but may be made even more difficult due to limited sampling, tissue fragmentation or tissue distortion. Common problems include the distinction of atypical ductal hyperplasia $(\mathrm{ADH})$ from limited examples of low grade ductal carcinoma in situ (DCIS); identifying foci of microinvasion in association with DCIS; distinguishing between DCIS and lobular carcinoma in situ (LCIS); distinguishing tubular carcinoma and low grade invasive ductal carcinoma from microglandular adenosis, sclerosing adenosis and other benign sclerosing lesions; distinguishing mucocele-like lesions from mucinous carcinoma; and the evaluation of columnar cell lesions, papillary lesions, spindle cell lesions, fibroepithelial lesions, and vascular lesions. Several guiding principles are relevant for all of these differential diagnostic issues and problematic areas in CNB specimens. First, the pathologist should always be aware of the radiologist's differential diagnosis and level of suspicion. If this information is not provided on the pathology requisition, it should be sought in the medical record or directly from the radiologist. Interpretation of CNB specimens without knowledge of the clinical and radiographic findings is strongly discouraged and could lead to erroneous or incomplete interpretations. Second, the pathologist should have a low threshold for obtaining additional levels if the pathologic findings on the initial sections do not correlate with findings on the imaging studies. Additional levels are also frequently useful for demonstrating histologic features that permit the correct diagnosis in challenging lesions. Immunostains should be used when necessary to help resolve differential diagnostic dilemmas, but these stains should be used judiciously and appropriately. Finally, it is most prudent to err on the conservative side when rendering diagnoses on CNB specimens with uncertain or equivocal findings; overdiagnosis should be avoided. If it is not possible to render a specific, unequivocal diagnosis, the diagnosis or differential diagnosis provided should be one that is sufficient to get the patient to the appropriate next step for evaluation or management.

There are several potentially important diagnostic pitfalls that the pathologist should keep in mind when interpreting breast CNBs. First, not all carcinomas encountered in a breast CNB represent primary breast cancers. A variety of extramammary malignancies may metastasize to the breast and may be erroneously interpreted as primary breast carcinomas. Features of carcinomas in CNB specimens that should raise concern for a metastatic lesion rather than a breast primary tumor include the absence of an in situ component, a histologic appearance that is unusual for a breast primary, the presence of extensive lymphovascular invasion with little or no associated stromal invasion, and a history of a prior or concurrent carcinoma at another site. In some cases, an appropriate panel of immunostains may be necessary to determine if a lesion is likely to be a primary breast carcinoma 


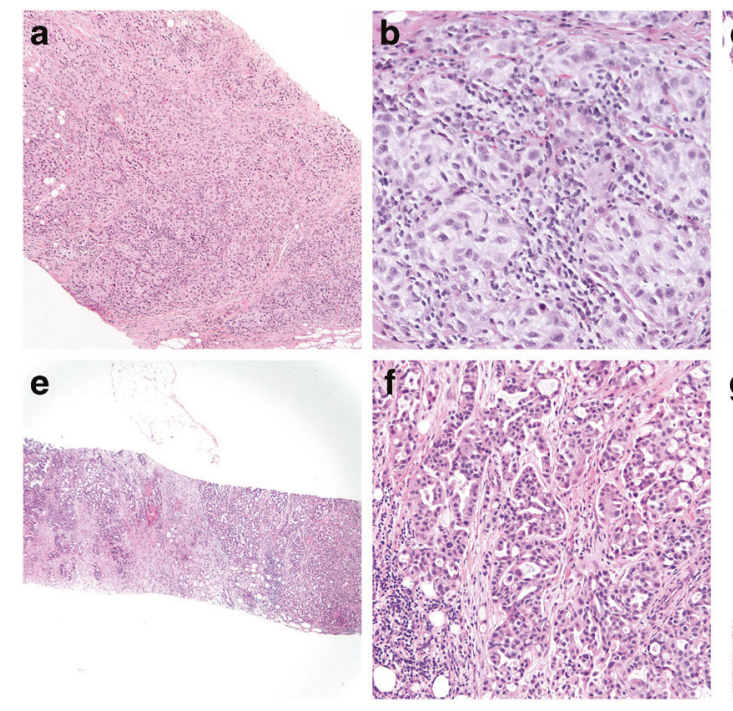

Fig. 1 Examples of tumors with epithelioid or epithelial features we have encountered in breast core needle biopsies that were intially mistaken for primary breast carcinomas: Metastatic malignant
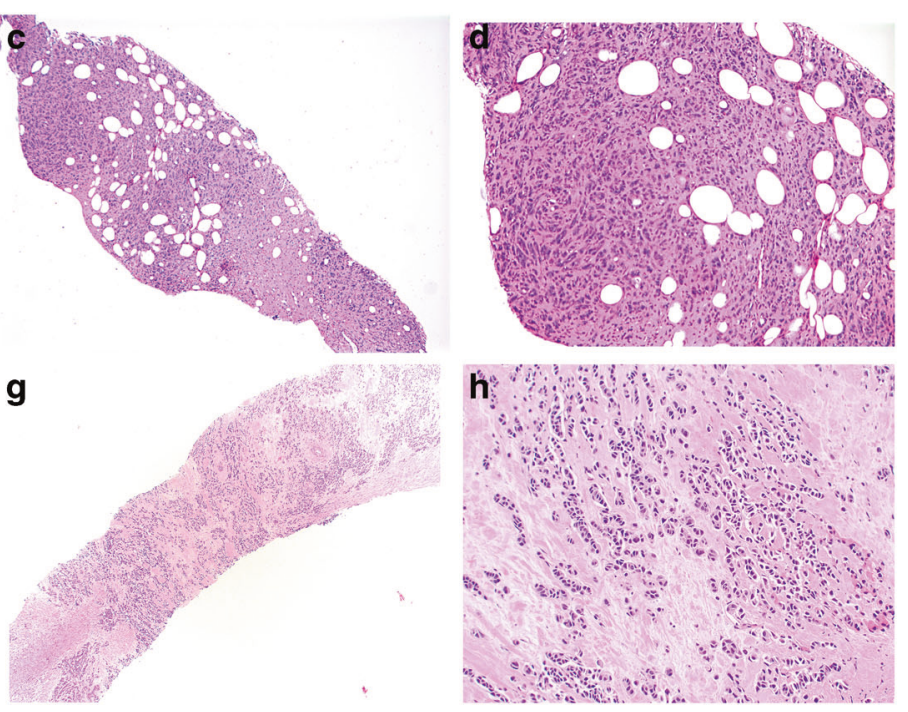

melanoma (a, b); post-radiation epithelioid angiosarcoma (c, d); metastatic lung adenocarcinoma (e, f); and metatastic medullary thyroid carcinoma $(\mathbf{g}, \mathbf{h})$ or a metastasis from another site. Second, not all epithelioid malignancies in a breast CNB represent carcinomas. Tumors with epithelioid histologic features that may mimic primary breast carcinomas include malignant melanoma, epithelioid angiosarcoma, and anaplastic large cell lymphoma, among others (Fig. 1). Finally, not all lesions present in breast CNB represent lesions of the breast parenchyma. CNB specimens that sample lesions of the subcutaneous adipose tissue of the breast (e.g., angiolipomas) and even the breast skin (e.g., epidermal inclusion cysts, dermatofibromas) are sometimes submitted as "breast lesions".

\section{Management of high risk lesions identified in core needle biopsies}

High risk breast lesions are those associated with an increased risk for the subsequent development of breast cancer based on the results of long-term follow-up studies. These include ADH, atypical lobular hyperplasia (ALH), LCIS, and DCIS. In addition to the concern about future breast cancer risk, the more immediate concern when these lesions are encountered in a CNB is the potential for the presence of a concurrent "worse" lesion missed by $\mathrm{CNB}$ and requiring treatment at that time. Thus, if $\mathrm{ADH}, \mathrm{ALH}$, or LCIS is seen in a CNB specimen, there is concern for concurrent DCIS or invasive breast cancer; if DCIS is identified in a CNB, there is concern for concurrent invasive carcinoma. Whether or not surgical excision is recommended for any lesion detected on CNB depends upon the frequency of "upgrade" to a worse lesion on subsequent surgical excision. A large number studies published over the past two decades has addressed the issue of upgrade rates of high risk lesions found in CNB specimens [6]. Unfortunately, many of these studies have one or more limitations including small numbers of cases, variation in guidance methods and biopsy devices used between and even within studies, lack of central pathology review, and lack of information on radiologic-pathologic concordance or even radiologic-pathologic discordance in some cases. In addition, most studies have been retrospective, and in some studies not all patients underwent excision leading to a concern about selection bias.

\section{Atypical ductal hyperplasia}

In a recent literature review of 42 studies, upgrade rates at surgical excision for ADH on CNB ranged from $0 \%$ to $62 \%$ with a mean of $23 \%$ [7]. In that review an upgrade to DCIS was about five times more likely than an upgrade to invasive cancer. Higher upgrade rates have been associated with a variety of mamographic features (mass, linear branching calcifications), technical factors (smaller gauge needles, no vacuum assistance, incomplete removal of the mammogrpahic lesion), and pathologic features (more extensive lesions/greater number of foci, specific histologic features such as a micropapillary pattern and features that border on DCIS). Unfortunately the association between these various mammographic, technical, and pathologic features, and upgrade rates have not been consistently observed in all studies. For example, in one study of 101 cases of ADH diagnosed on 9 or 11 gauge, vacuum-assisted biopsies, even among cases with small lesions (i.e., $<3$ foci of $\mathrm{ADH}$ ) and 
complete removal of mammographic microcalcifications, the upgrade rate was still 12\% [8]. Nonetheless, identification of patients with ADH on CNB who can be spared excision remains an area of active research. Investigtors at M.D. Anderson Cancer Center recently reported on a series of 125 women with $\mathrm{ADH}$ on $\mathrm{CNB}$ who were managed with follow-up rather than surgical excision. Patients in this study had $<3$ foci of ADH, no mass on imaging studies and $>50 \%$ of the mammographic microcalcifications removed. With a median follow-up of 3 years, breast cancer was detected in $5 \%$ of patients [9].

In summary, given that we still cannot reproducibly identify which ADH lesions are unlikely to be associated with a worse lesion at the time of the $\mathrm{CNB}$, routine surgical excision is recommended for these patients

(https://www.breastsurgeons.org/new_layout/about/sta tements/PDF_Statements/Concordance_and_HighRiskLesions. pdf).

\section{Atypical lobular hyperplasia and lobular carcinoma in situ}

A recent literature review of 26 studies noted upgrade rates at surgical excision for ALH on CNB ranging from $0 \%$ to $67 \%$ with a mean of $9 \%$. In that same review, a mean upgrade rate of $18 \%$ (range $0-60 \%$ ) was noted in 29 studies of LCIS [7]. However, given the interobserver variability in the distinction between ALH and LCIS, lack of central pathology review in some published studies, and similar clinical management for ALH and LCIS, for the purposes of this discussion it is best to consider these lesions together under the heading of "lobular neoplasia". Recent studies of lobular neoplasia on core biopsy in which the lesion was incidental finding and there were concordant imaging findings have consistently shown a low frequency of upgrades at surgical excision, ranging from $0 \%$ to $3 \%$ [10]. These more recent data have led many institutions to offer patients with lobular neoplasia on CNB and concordant imaging findings the option of follow-up without surgical excision [6]. This approach is currently supported by the National Comprehensive Cancer Network (NCCN) guidelines as well as a guideline published by the American Society of Breast Surgeons (ASBS) (https://www.brea stsurgeons.org/new_layout/about/statements/PDF_Sta tements/Concordance_and_HighRiskLesions.pdf). In contrast, the upgrade rate for pleomorphic LCIS identified on CNB has been reported to be $17-46 \%$. Therefore, surgical excision of such lesions is required [11-15].

\section{Ductal carcinoma in situ}

Until recently, it was simply accepted as a limitation of CNB that some cases of DCIS would be upgraded to invasive carcinoma at excision. In a meta-analysis of 52 studies that included 7350 cases of DCIS on CNB followed by surgical excision, the upgrade rate was $25.9 \%$ [16]. Therefore surgical excision has traditionally been considered mandatory for these patients. While there are now several clinical trials being conducted around the world evaluating the role of active surveillance without surgical excison for selected women with DCIS detected on CNB (COMET, LORD, LORIS) [17], in routine clinical practice surgical excision is required for a diagnosis of DCIS on CNB.

A summary of current recommendations for the management of high risk lesions detected on CNB is presented in pathologic features extracted from pathology reports (diagnosis and descriptive text) to predict upgrade rates in women with high risk lesions identified on CNB is presented in Table 1.

A recent study reported the development of a machine learning algorithm based on clinical features and pathologic features extracted from pathology reports (diagnosis and descriptive text) to predict upgrade rates in womenh with high risk lesions identifed on CNB. The model was developed with 667 high risk lesions and then tested on an independent set of 335 high risk lesions. In this study, if patients categorized by the model as being at low risk of upgrade had not been excised, only one of 38 cancers (2.6\%) would have been missed and $31 \%$ of patients would have been spared excision [18]. The use of artificial intelligence and machine learning to evaluate and integrate clinical, mammographic, and pathologic features to more accurately predict upgrade rates for women with high risk lesions on CNB merits further evaluation.

\section{Ancillary biomarker and molecular testing in breast core needle biopsies}

In current clinical practice, the only biomarker assays that should be performed routinely on breast CNBs are estrogen receptor (ER) and progesterone receptor (PR) immunhistochemistry and HER2 immunohistochemistry and/or FISH assays for cases of invasive breast cancer and ER (with or without PR) assays for cases of DCIS [19]. However, a recent study questioned the cost-effectiveness of performing ER (and PR) assays on CNB specimens with DCIS and suggested deferring biomarker assays in such cases to the subsequent surgical specimen [20]. There is a randomized clinical trial evaluating the role of trastuzumab in patients with HER2-positive DCIS (NSABP B-43); however, there is currently no role for routine HER2 testing of DCIS in clinical practice. Occasionally the 21 -gene recurrence score 
assay (OncotypeDX) is performed on CNB specimens to determine the most suitable neoadjuvant systemic therapy. However, routine testing for somatic mutations in cancer genes, as has become common practice on other tumor types, is not currently performed on CNB of newly diagnosed breast cancers.

\section{Compliance with ethical standards}

Conflict of interest The author declares that he has no conflict of interest.

\section{References}

1. Bilous M. Breast core needle biopsy: issues and controversies. Mod Pathol. 2010;23(Suppl 2):S36-45.

2. O'Flynn EA, Wilson AR, Michell MJ. Image-guided breast biopsy: state-of-the-art. Clin Radiol. 2010;65:259-70.

3. Eby PR, Lehman CD. Magnetic resonance imaging--guided breast interventions. Top Magn Reson Imaging. 2008;19:151-62.

4. Ibrahim RE, Sciotto CG, Weidner N. Pseudoangiomatous hyperplasia of mammary stroma. Some observations regarding its clinicopathologic spectrum. Cancer. 1989;63:1154-60.

5. Rosa G, Dawson A, Rowe JJ. Does identifying whether pseudoangiomatous stromal hyperplasia (PASH) is focal or diffuse on core biopsy correlate with a PASH nodule on excision? Int J Surg Pathol. 2017;25:292-7.

6. Calhoun BC. Core needle biopsy of the breast: an evaluation of contemporary data. Surg Pathol Clin. 2018;11:1-16.

7. Mooney KL, Bassett LW, Apple SK. Upgrade rates of high-risk breast lesions diagnosed on core needle biopsy: a single-institution experience and literature review. Mod Pathol. 2016;29:1471-84.

8. Kohr JR, Eby PR, Allison KH, DeMartini WB, Gutierrez RL, Peacock S, et al. Risk of upgrade of atypical ductal hyperplasia after stereotactic breast biopsy: effects of number of foci and complete removal of calcifications. Radiology. 2010;255: 723-30.

9. Menen RS, Ganesan N, Bevers T, Ying J, Coyne R, Lane D, et al. Long-term safety of observation in selected women following core biopsy diagnosis of atypical ductal hyperplasia. Ann Surg Oncol. 2017;24:70-6.

10. Nakhlis F, Gilmore L, Gelman R, Bedrosian I, Ludwig K, Hwang ES, et al. Incidence of adjacent synchronous invasive carcinoma and/or ductal carcinoma in-situ in patients with lobular neoplasia on core biopsy: results from a Prospective Multi-Institutional Registry (TBCRC 020). Ann Surg Oncol. 2016;23:722-8.

11. Hwang H, Barke LD, Mendelson EB, Susnik B. Atypical lobular hyperplasia and classic lobular carcinoma in situ in core biopsy specimens: routine excision is not necessary. Mod Pathol. 2008;21:1208-16.

12. Niell B, Specht M, Gerade B, Rafferty E. Is excisional biopsy required after a breast core biopsy yields lobular neoplasia? Am J Roentgenol. 2012;199:929-35.

13. Atkins KA, Cohen MA, Nicholson B, Rao S. Atypical lobular hyperplasia and lobular carcinoma in situ at core breast biopsy: use of careful radiologic-pathologic correlation to recommend excision or observation. Radiology. 2013;269:340-7.

14. De Brot M, Koslow Mautner S, Muhsen S, Andrade VP, Mamtani A, Murray M, et al. Pleomorphic lobular carcinoma in situ of the breast: a single institution experience with clinical follow-up and centralized pathology review. Breast Cancer Res Treat. 2017;165:411-20. 
15. Masannat YA, Husain E, Roylance R, Heys SD, Carder PJ, Ali H, et al. Pleomorphic LCIS what do we know? A UK multicenter audit of pleomorphic lobular carcinoma in situ. Breast. 2018;38:120-4.

16. Brennan ME, Turner RM, Ciatto S, Marinovich ML, French JR, Macaskill P, et al. Ductal carcinoma in situ at core-needle biopsy: meta-analysis of underestimation and predictors of invasive breast cancer. Radiology. 2011;260:119-28.

17. Grimm LJ, Ryser MD, Partridge AH, Thompson AM, Thomas JS, Wesseling J, et al. Surgical upstaging rates for vacuum assisted biopsy proven DCIS: implications for active surveillance trials. Ann Surg Oncol. 2017;24:3534-40.

18. Bahl M, Barzilay R, Yedidia AB, Locascio NJ, Yu L, Lehman CD. High-risk breast lesions: a machine learning model to predict pathologic upgrade and reduce unnecessary surgical excision. Radiology. 2018;286:810-8.

19. Allred DC, Anderson SJ, Paik S, Wickerham DL, Nagtegaal ID, Swain SM, et al. Adjuvant tamoxifen reduces subsequent breast cancer in women with estrogen receptor-positive ductal carcinoma in situ: a study based on NSABP Protocol B-24. J Clin Oncol. 2012;30:1268-1273.

20. VandenBussche CJ, Cimino-Mathews A, Park BH, Emens LA, Tsangaris TN, Argani P. Reflex estrogen receptor (ER) and progesterone receptor (PR) analysis of ductal carcinoma in situ (DCIS) in breast needle core biopsy specimens: an unnecessary exercise that costs the United States \$35 million/y. Am J Surg Pathol. 2016;40:1090-9. 